\title{
A quantitative risk analysis method for the natural gas pipeline network
}

\author{
Y.-D. Jo ${ }^{1}$, K.-S. Park ${ }^{1}$, H.-S. Kim ${ }^{1}$, J.-J. Kim ${ }^{1}$, J.-Y. Kim ${ }^{2}$ \\ \& J. W. $\mathrm{Ko}^{3}$ \\ ${ }^{1}$ Institute of Gas Safety Technology, Korea Gas Safety Corporation, \\ Republic of Korea \\ ${ }^{2}$ Department of Mechanical Engineering, Chung-Ang University, \\ Republic of Korea \\ ${ }^{3}$ Department of Chemical Engineering, Kwangwoon University, \\ Republic of Korea
}

\begin{abstract}
Natural gas as a fuel is supplied in the most populous urban areas with the risk threatening public safety managed properly. Regulatory authorities in many countries are moving away from prescriptive approaches for keeping natural gas pipelines safe. As an alternative, risk management based on a quantitative assessment is considered to improve the level of safety. This work focuses on the quantitative risk assessment for the natural gas pipelines network and introduces parameters of fatal length and cumulative fatal length. The fatal length is defined as the integrated fatality along the pipeline associated with hypothetical accidents. The cumulative fatal length is defined as the length of pipeline section in which an accident leads to $\mathrm{N}$ or more fatalities. These parameters can be calculated easily within a geographic information system (GIS). For a new gas pipeline that will be installed in a suburban area, the risks are estimated simply in a few hours by using this method. This quantitative risk assessment may be useful for risk management during the planning and building stages of a new pipeline, modification of the pipeline, and to lower the risk of a buried pipeline network.
\end{abstract}

Keywords: risk analysis, gas pipeline network, individual risk, societal risk, jet fire. 


\section{Introduction}

High-pressure pipelines carrying natural gas are not on secure industrial sites, as a potentially hazardous plant would be, but are routed across the land i.e., a busy city or a network of superhighways. Consequently, there is the ever-present potential for third parties to interfere with the integrity of these pipelines. In addition, the combination of third-party interference and pipeline route might suggest that people around the pipelines are subject to significant risk from pipeline failure. If the natural gas is accidentally released and ignited, the hazard distance associated with these pipelines to people and property is known to range from under $20 \mathrm{~m}$ for a smaller pipeline at lower pressure, up to over $300 \mathrm{~m}$ for a larger one at higher pressure [1]. Therefore, pipeline operators and regulators must address the associated public safety issues.

The network of high-pressure natural gas pipelines is inevitable to supply the gas as a fuel to houses in the Seoul metropolitan area, due to mismatch between gas supply and demand when the gas pressure is reduced at this moment. Therefore, the risk management of high-pressure natural gas pipelines is to be issued until the pipeline is moved into a low populated area or the operating pressure of the pipeline is reduced.

This paper focuses on a simplified method to calculate explicitly the risk of a high-pressured natural gas pipeline network with reasonable accident scenarios.

\section{Gas release rate from gas pipeline}

The gas release rate from a hole in the gas pipeline varies with time. Within seconds of failure, the release rate will have dropped to a fraction of the peak initial value. It will decay even further over time until steady-state. The peak initial release can be estimated by assuming the sonic flow through an orifice as in the following eqn. [2].

$$
Q_{\text {peak }}=\frac{\pi d^{2} \alpha}{4} \sqrt{\gamma \rho_{0} p_{0}\left[\frac{2}{\gamma+1}\right]^{\frac{\gamma+1}{\gamma-1}}}
$$

where $\alpha$ is the dimensionless hole size, which is the ratio of effective hole area to the pipe cross-sectional area, $d$ is the pipeline diameter, $\rho_{0}$ is the stagnation density of gas at operating conditions, $p_{0}$ is stagnation pressure at operating conditions, and $\gamma$ is the specific heat ratio of gas.

The release rate through a hole in the pipeline at steady-state can be estimated approximately with eqn. (1) [3].

$$
Q_{\text {steady-state }}=\frac{Q_{\text {peak }}}{\sqrt{1+\frac{4 \alpha^{2} f_{F} L}{d}\left(\frac{2}{\gamma+1}\right)^{\frac{2}{\gamma-1}}}}
$$

where $f_{F}$ is Fanning friction factor and $L$ is the pipeline length from the gas supply station to the release point. 
The numerator in the above equation is the release rate without friction loss through the pipeline, while the denominator acts as a decay factor due to the wall friction loss at steady-state. The effective release rate associated with the death probability of a person from a fire would depend on the time exactly when ignition occurs. The death probability could be estimated by approximating the transient jet fire as a steady-state fire that is fed by the gas released at the effective rate. The effective release rate, $C Q_{\text {peak }}$, is a fractional multiple of the peak initial release rate. It can be used to obtain the heat flux comparable to that from the real transient fire ignited in slight delay. In general, the most appropriate value for the decay factor would depend on the pipe size being considered, the pressure at the time of failure, the assumed time to ignition, and the time period required to cause harm to people. In one-dimensional transient flow through the arrested crack tip of a tube with constant cross-section, the decay factor is expressed as the following equation [4].

$$
C=\left[1-\frac{\gamma-1}{\gamma+1}\right]^{\frac{2 \gamma}{\gamma-1}}
$$

In a study of risks from hazardous pipelines in the UK conducted by A. D. Little Ltd. [5], the authors quoted 0.25 as the decay factor. A more conservative value of 0.3 is adopted here for the factor. It is not to underestimate the intensity of the sustained fire associated with nearly immediate ignition of leaked gas from large diameter pipelines. For a rupture near the gas supplying station, however, the decay factor appears at steady-state greater than 0.3 which is estimated by the denominator of eqn. (2). Therefore, the decay factor has to be taken the lager value between 0.3 and the value at steady-state [6].

By assuming the specific heat ratio $\gamma=1.42$, gas density at atmosphere $\rho=0.68 \mathrm{~kg} / \mathrm{m}^{3}$, and Fanning friction factor $f_{F}=0.0026$ conservatively for steel pipeline, the effective rate of gas release through a hole on the pipeline can be expressed as the following equation.

For an accident near the gas supplying station:

$$
Q_{e f f, i}=\frac{1.783 \times 10^{-3} A_{P} \alpha_{i} p_{0}}{\sqrt{1+4.196 \times 10^{-3} \alpha_{i}{ }^{2} L / d}}, \alpha_{i}{ }^{2} \frac{L}{d} \leq 2410
$$

For an accident far away from the gas supplying station:

$$
Q_{\text {eff }, i}=5.349 \times 10^{-4} A_{P} \alpha_{i} p_{0}, \alpha_{i}^{2} \frac{L}{d}>2410
$$

where $A_{p}$ is the cross-section of the pipeline.

To analysis a gas pipeline network, an equivalent straight pipeline length can be estimated by using the analogy of gas flow in a pipeline network to electron flow in electric circuit as shown in fig. 1. The denominator of eqn. (2) could be considered as the resister in the electric circuit. 


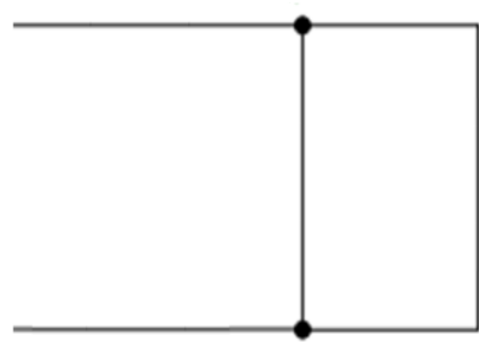

(a) Gas pipeline network

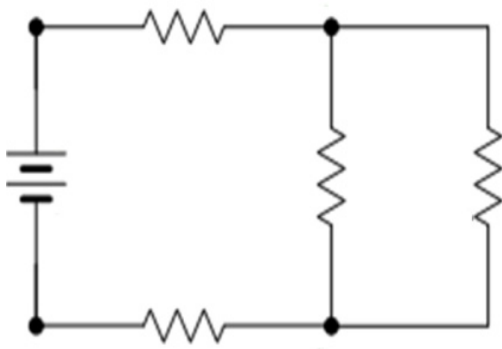

(b) Electric circuit

Figure 1: Analogy between gas pipeline network and electric circuit.

Table 1: $\quad$ Failure frequencies based on failure causes and hole size (EGIG, 1993).

\begin{tabular}{|c|c|c|c|c|c|}
\hline \multirow[t]{2}{*}{ Failure causes } & \multirow{2}{*}{$\begin{array}{c}\text { Failure } \\
\text { frequency } \\
{[1 / \mathrm{yr} . \mathrm{km}]}\end{array}$} & \multirow{2}{*}{$\begin{array}{c}\text { Percentage } \\
\text { of total } \\
\text { failure rate }\end{array}$} & \multicolumn{3}{|c|}{$\begin{array}{c}\text { Percentage of different } \\
\text { hole size }[\%]^{*}\end{array}$} \\
\hline & & & Small & Medium & Great \\
\hline External interference & $3.0 \times 10^{-4}$ & $51 \%$ & 25 & 56 & 19 \\
\hline Construction defects & $1.1 \times 10^{-4}$ & $19 \%$ & 69 & 25 & 6 \\
\hline Corrosion & $8.1 \times 10^{-5}$ & $14 \%$ & 97 & 3 & $<1$ \\
\hline Ground movement & $3.6 \times 10^{-5}$ & $6 \%$ & 29 & 31 & 40 \\
\hline Others/unknown & $5.4 \times 10^{-5}$ & $10 \%$ & 74 & 25 & $<1$ \\
\hline
\end{tabular}

$\begin{array}{llllll}\text { Total failure rate } & 5.75 \times 10^{-4} & 100 \% & 48 & 39 & 13\end{array}$

* The hole sizes are defined as follows: Small hole: hole size is lower than $2 \mathrm{~cm}$; Medium hole: hole size ranges from $2 \mathrm{~cm}$ up to the pipe diameter; Great hole: Full bore rupture or hole size is greater than the pipe diameter.

If the location of leak point is more than a few kilometres away from gas supplying station, the effective gas release rate is independent to the pipeline length and can be estimated simply by eqn. (5).

\section{Failure rate of gas pipeline}

The failure rate in a particular section of pipeline depends on many variables, such as soil, coating, design, cathodic protection, age of pipeline, depth of cover, hydrostatic test, survey, patrol, training, and so on. It is very difficult to include the effects of those variables on the failure rate because accident data may not be 
sufficient for statistical analysis. Generally for the risk analysis, the failure rate of pipeline is estimated simply by using some variables from historical data. The failure rate of major gas pipelines in Western Europe is reported by the European Gas Pipeline Incident Data Group (EGIG) [7]. It is currently based on the experience of 1.5 million kilometre-years in eight countries of Western Europe. As shown in table 1, the external interference by third party activity is the leading cause to major accidents with the medium or great hole generated. The total failure rates for small, medium and great hole are $2.76 \times 10^{-4}, 2.243 \times 10^{-4}$ and $7.475 \times 10^{-5} 1 / \mathrm{yr} . \mathrm{km}$, respectively. These values are an order of magnitude higher than the values estimated from DOT data or British Gas Transco data [8]. In this work, we adapt the EGIG data conservatively.

\section{Method of quantitative risk assessment}

The risk can be evaluated from the two quantities: the probability that a failure of the pipeline can occur and the consequences that arises as the result. The failure rate of a pipeline section may be estimated by adding the failure rates caused by external interference, construction defect, corrosion, ground movement and unknown. The rate caused by external interference can be estimated by using BG Transco data and that by other causes can be estimated simply by using EGIG data.

By defining the fatal length as the integrated fatality along the pipeline associated with hypothetical accidents, the individual risk at a specified location can be expressed simply as following equation.

$$
I R=\sum_{i} L_{F L, i} \varphi_{i}
$$

where $\varphi_{i}$ failure rate per unit length of the pipeline associated with the accident scenario $i$.

The fatal length can be approximated simply by adding the pipe lengths multiplied with corresponding average values of lethality in the zones of $1 \%$ $50 \%, 50-99 \%$, and $99 \%-100 \%$ lethality. These zones are divided by drawing circle of radii $99 \%, 50 \%, 1 \%$ fatality with a specified location as origin. The dominant hazard from natural gas pipeline is jet fire [9]. By analyzing the damage to people from the fire of natural gas, these radii are:

$$
r_{99}=\sqrt{15.3 Q_{e f f}} ; \quad r_{50}=\sqrt{30.4 Q_{e f f}} ; \quad r_{i}=\sqrt{60.3 Q_{e f f}}
$$

By using the eqns. (4), (5) and (7), and the average fatalities of those three zones as $1,0.86,0.156$, respectively, the fatal length at specified location can be estimated by the length of pipeline within each zone as following equation.

$$
L_{F L} \approx l_{100-99}+0.86 l_{99-50}+0.156 l_{50-1}
$$


Even though the approximate solution has some deviation from the exact one, it has a big advantage of being extended easily to the curved gas pipeline as well as being estimated directly within a Geographic Information System (GIS).

The societal risk is defined from the societal point of view. It is expressed with the cumulative frequency and the expected number of death caused by an accident. The expected number of death from a hypothetical accident could be calculated by integrating the multiplication of fatality and population density within hazard area.

The number of fatalities from an accident may be calculated simply by considering the number of persons and by assuming an average probability of death within the area encountered. As discussed in individual risk, the area can be divided also into three zones of $1 \%-50 \%, 50 \%-99 \%$, and $99 \%-100 \%$ lethality. The number of fatalities from an accident can be estimated approximately as following equation.

$$
N=N_{100-99}+0.802 N_{99-50}+0.142 N_{50-1}
$$

To take the discrete hazardous sources into consideration, a pipeline should be divided into small sections. It should be short enough not to influence the calculated results. A profile can be drawn up graphically with thus calculated fatalities from an accident at each pipe segment. The curve could be constructed in a manner of segment by segment over the entire pipeline. It takes generally the shape of a ball. The cumulative fatal length is determined simply from the profile of fatalities. It is just the length of the horizontal line of fatalities $\mathrm{N}$ intersected by the fatality curve. By assuming constant failure rate within the pipeline, the societal risk can be expressed with the cumulative fatal length [6].

$$
F=\sum_{i} \varphi_{i} L_{C F L, i}\left(N_{i} \geq N\right)
$$

The cumulative fatal length, $L_{C F L}$, means the length of pipeline within which an accident leads to $\mathrm{N}$ or more fatalities.

\section{Risk calculation}

Two types of risk, individual risk and societal risk, are estimated by using the method presented in the above section, before a gas pipeline of $300 \mathrm{~mm}$ diameter, operating at $30 \mathrm{bar}$, covered $150 \mathrm{~cm}$ depth is to be constructed in a suburban area where the average population density is $2.8 \times 10^{-3}$ person $/ \mathrm{m}^{3}$ as shown in fig. 2. From eqns. (5), (6), and (8), the fatal length is proportional to the square root of the effective gas release rate and the release rate is proportional to the operation pressure. Therefore, the individual risk is proportional to the square root of the operating pressure of the pipeline. 

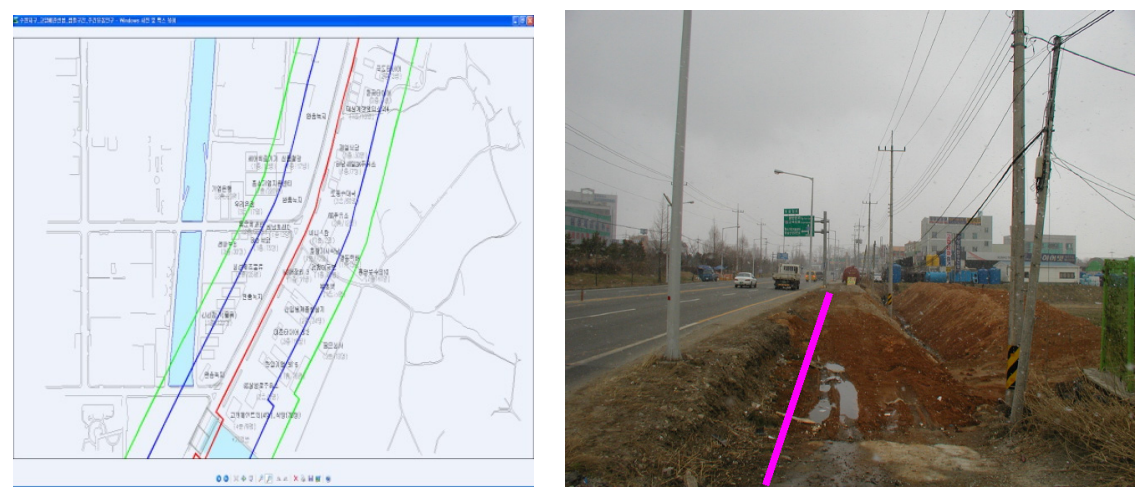

Figure 2: $\quad$ A natural gas pipeline planned to be located in a suburban area.

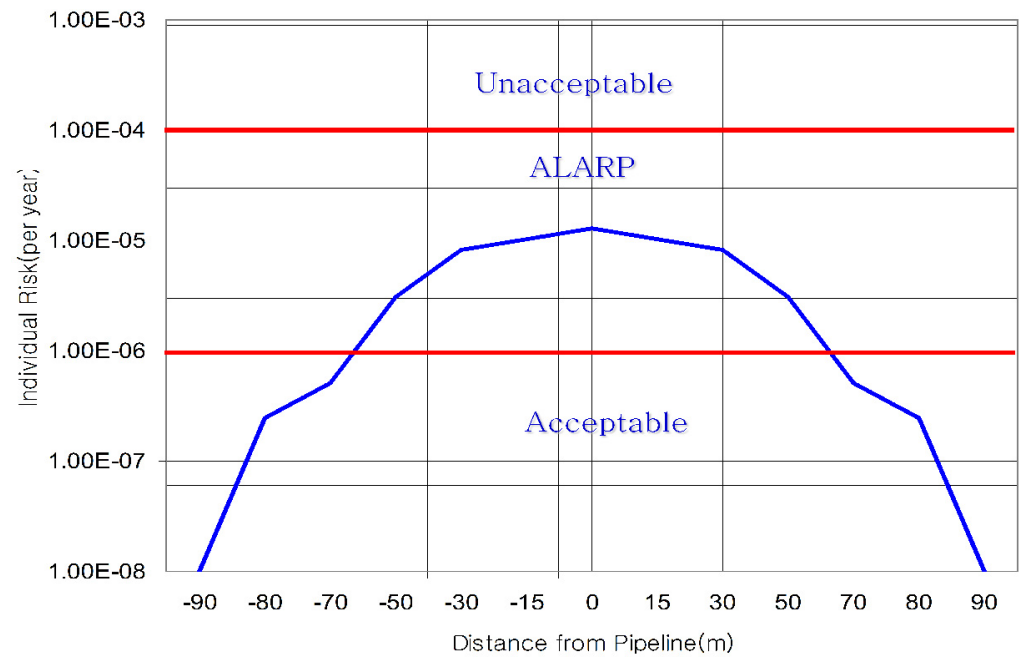

Figure 3: Individual risk at a distance from the gas pipeline.

The individual risk experienced by a single individual in a year is lower than $10^{-6} / \mathrm{yr}$ when the proximity distance to the pipeline is greater than $60 \mathrm{~m}$ as shown in fig. 3. In the horizontal axis of fig. 3, the minus and positive sign means the left and right side from the gas pipeline. A place located within $60 \mathrm{~m}$ from the pipeline, the individual risk is located in ALARP [as low as reasonably practicable] zone. The societal risk is also in ALARP zone as shown in fig. 4. To install the gas pipeline, the owner of the pipeline has to implement risk reduction measures as far as possible. 


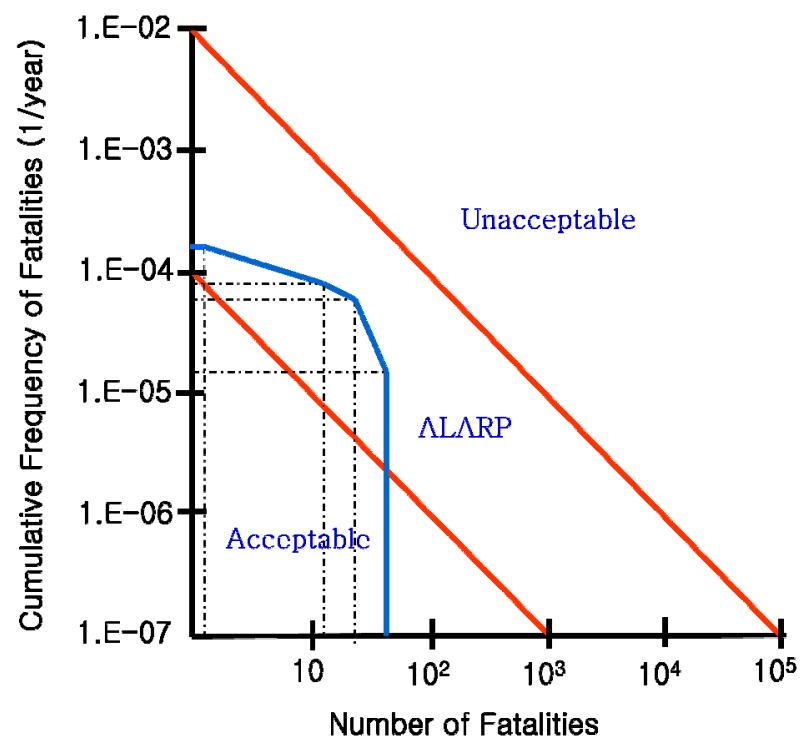

Figure 4: $\quad$ Societal risk of the gas pipeline.

The quantitative risk, individual risk and societal risk, of high-pressure natural gas pipeline network can be simply estimated by using the fatal length and the cumulative fatal length with EGIG failure data. This method can be very useful to estimate the risk in a few hours.

\section{Conclusions}

A simple method of quantitative risk assessment is proposed with new parameters, i.e., the fatal length and the cumulative fatal length. These parameters are estimated directly within a Geographic Information System (GIS) and are sensitive to pipeline length, pipeline diameter, and operating conditions. The proposed method turns out to be successful through being applied to calculate the risk for a high-pressure pipeline. The individual risk is proportional to the square root of the operating pressure of the pipeline. This method of quantitative risk assessment may be useful for risk management during the planning and building stages of a new pipeline, modification of the pipeline, and to lower the risk of a buried pipeline network.

\section{References}

[1] Jo, Y.-D., \& Ahn, B.J., Analysis of hazard area associated with highpressure natural-gas pipeline, Journal of Loss Prevention in the Process Industries, 15, pp. 179-188, 2002. 
[2] Crowl, D.A. \& Louvar, J.F., Chemical Process Safety Fundamentals with Applications (2 ${ }^{\text {nd }}$ ed.). New Jersey: Prentice-Hall, 2002, p. 100.

[3] Jo, Y.-D. \& Ahn, B.J., A simple model for the release rate of hazardous gas from a hole on high pressure pipeline. J. of Hazardous Materials, 97(1-3), pp. 31-46, 2003.

[4] Bragaw, C.G., Rapid crack propagation in MDPE. Proc. $7^{\text {th }}$ Symp. Plastic Fuel Gas Pipe (J1-J31). American Gas Association, New Orleans, U.S., November, 1979.

[5] Hill, R.T. \& Catmur, J.R., Risks from hazardous pipelines in the United Kingdom. Health and Safety Executive Contract Research Report No. 82/1994, 1994.

[6] Jo, Y.-D., \& Ahn, B.J., A method of quantitative risk assessment for transmission pipeline carrying natural gas. J. of Hazardous Materials, A123,pp. 1-12, 2005.

[7] European Gas Pipeline Incident Data Group, Gas Pipeline Incidents $4^{\text {th }}$ Report 1970-1998, 1999.

[8] John, M., Chris, B., Andrew, P., \& Charlotte, T., An Assessment of Measures in Use for Gas Pipeline to Mitigate against Damage Caused by Third Party Activity, Printed and Published by the Health and Safety Executive, C1 10/01, 2001.

[9] Jo, Y.-D. \& Crowl, D. A., Individual risk analysis of high-pressure natural gas pipelines. Journal of Loss Prevention in the Process Industries, 21, pp. 589-595, 2008. 